\section{PTH-002 A META-ANALYSIS OF ENDOSCOPIC ULTRASOUND WITH FINE NEEDLE ASPIRATION (EUS-FNA) FOR DIAGNOSIS OF SOLID PANCREATIC NEOPLASMS}

doi:10.1136/gut.2011.239301.403

M Hewitt, $1,{ }^{1}$ M McPhail, ${ }^{2}$ L Possamai, ${ }^{2}$ P Vlavianos, ${ }^{2}$ A Dhar, ${ }^{2} \mathrm{~K}$ Monahan ${ }^{3}{ }^{1} / \mathrm{mperial}$ College, London, UK; ${ }^{2}$ Gastroenterology, Imperial College Healthcare Trust, London, UK; ${ }^{3}$ Gastroenterology, West Middlesex University Hospital, London, UK

Introduction Pancreatic cancer has one of the lowest 5-year survival rates of all cancers. Median survival postdiagnosis is 6 months and so early and accurate diagnosis is vital to improve chance of survival. EUS-FNA has the benefit of being a minimally invasive, well-tolerated procedure although results are operator dependent. The aim of this study is to determine the diagnostic accuracy of EUS-FNA for pancreatic cancer via a meta-analysis of published studies.

Methods Relevant studies were identified via MEDLINE and included if they used a reference standard of definitive surgical histology or clinical follow-up of at least 6 months. Study quality was assessed using the STARD (STAndards for the Reporting of Diagnostic Accuracy) initiative criteria with a 25-point STARD checklist. Data was analysed by dichotomous classification; where cytology was considered suspicious or atypical this was considered negative for malignancy (Classification \#1) or positive for malignancy (Classification \#2). Heterogeneity was explored via the I2 statistic and subgroup analysis was performed using the following variables: publication year, study location, number of patients, number of centres involved, study design (retrospective/prospective) and length.

Results 33 studies published between 1997 and 2009 were included. 12 were retrospective studies and 21 prospective. The median number of patients included in each study was 151

Table 1 PTH-002

\begin{tabular}{|c|c|c|c|c|c|c|}
\hline \multirow[b]{2}{*}{ Subgroup } & \multirow[b]{2}{*}{ Studies (N) } & \multicolumn{2}{|c|}{ Sensitivity } & \multicolumn{2}{|c|}{ Specificity } & \multirow[b]{2}{*}{ AUC } \\
\hline & & Pooled & I-Sq & Pooled & I-Sq & \\
\hline All studies (\#1) & 33 & 0.85 & 82.1 & 0.98 & 53.2 & 0.95 \\
\hline All studies (\#2) & 33 & 0.91 & 72.6 & 0.94 & 59.4 & 0.96 \\
\hline \multicolumn{7}{|l|}{ Study design } \\
\hline Retrospective & 12 & 0.8 & 82.8 & 0.99 & 0 & 0.94 \\
\hline Prospective & 22 & 0.87 & 78.3 & 0.98 & 65.3 & 0.94 \\
\hline \multicolumn{7}{|l|}{ STARD } \\
\hline$>13<19$ & 27 & 0.86 & 83 & 0.97 & 58.5 & 0.94 \\
\hline$\geq 19$ & 7 & 0.82 & 73.8 & 0.99 & 0 & 0.97 \\
\hline \multicolumn{7}{|l|}{ Number of centres } \\
\hline Single & 29 & 0.84 & 82.4 & 0.97 & 51 & 0.95 \\
\hline Multi & 5 & 0.99 & 47.9 & 0.87 & 79.7 & 0.98 \\
\hline
\end{tabular}


(range 27-557) and the median study length was 44 months (range 11-119). Based on classification \#1, the pooled sensitivity was 0.85 (95\% CI 0.84 to 0.86 ) and pooled specificity was 0.98 (95\% CI 0.97 to 0.99 ). When suspicious/atypical cytology was evaluated as positive for malignancy, it had an improved sensitivity of 0.91 and decreased specificity of 0.94 . Specificity was also higher in prospective and multicentred studies. The area under the ROC curve was 0.95 . Heterogeneity was decreased in subgroups considering only high quality studies.

Conclusion The meta-analysis shows that EUS-FNA is an excellent diagnostic test for solid neoplasms of the pancreas with a sensitivity of 0.85 (95\% CI 0.84 to 0.86 ) and a specificity of 0.98 (95\% CI 0.97 to 0.99 ), with an area under the ROC curve of 0.95 . EUS-FNA is a suitable first investigation to perform once a solid lesion is detected.

Competing interests None.

Keywords endoscopic ultrasound, fine needle aspiration, pancreatic cancer. 